\title{
Defense and adaptation: the complex inter-relationship between Campylobacter jejuni and mucus
}

\author{
Abofu Alemka ${ }^{1}$, Nicolae Corcionivoschi ${ }^{2}$ and Billy Bourke ${ }^{2,3 *}$ \\ ${ }^{1}$ Department of Biological Sciences, University of Alberta, Edmonton, AB, Canada \\ ${ }^{2}$ The National Children's Research Centre, Our Lady's Children's Hospital, Crumlin, Dublin, Ireland \\ ${ }^{3}$ Alimentary Glycosciences Research Group, School of Medicine and Medical Science, Conway Institute, University College Dublin, Dublin, Ireland
}

Edited by:

Alain Stintzi, Ottawa Institute of

Systems Biology, Canada

\section{Reviewed by:}

Charlene Kahler, University of

Western Australia, Australia

Stuart A. Thompson, Medical College

of Georgia, USA

\section{*Correspondence:}

Billy Bourke, The National Children's

Research Centre, Our Ladys

Children's Hospital, Crumlin, Dublin

12, Ireland.

e-mail: billy.bourke@ucd.ie
Mucus colonization is an essential early step toward establishing successful infection and disease by mucosal pathogens. There is an emerging literature implicating specific mucin sub-types and mucin modifications in protecting the host from Campylobacter jejuni infection. However, mucosal pathogens have evolved sophisticated mechanisms to breach the mucus layer and $C$. jejuni in particular appears to harbor specific adaptations to better colonize intestinal mucus. For example, components of mucus are chemotactic for $C$. jejuni and the rheological properties of mucus promote motility of the organism. Furthermore, recent studies demonstrate that mucins modulate the pathogenicity of $C$. jejuni in a species-specific manner and likely help determine whether these bacteria become pathogenic (as in humans), or adopt a commensal mode of existence (as in chickens and other animals). This review focuses on recent advances in understanding the complex interplay between $C$. jejuni and components of the mucus layer.

Keywords: Campylobacter, mucus, mucins, pathogenicity, motility, chickens

\section{INTRODUCTION} MUCUS COMPOSITION

The visco-elastic mucus gel layer consists of an outer loose layer containing gut microbiota and an inner more compact layer devoid of any bacteria (Johansson et al., 2008). The thickness of the mucus layer ranges from $10 \mu \mathrm{m}$ in the eye to $700 \mu \mathrm{m}$ in the intestine (Linden et al., 2008b). Mucin glycoproteins are the main components of mucus while others include; cathelicidins, defensins, lysozyme, protegrins, collectins, antibodies, nitric oxide, and histatins. Collectively these constituents of mucus form a physical barrier, participate in bacterial clearance, and display antimicrobial activity (Linden et al., 2008b; McGuckin et al., 2011). Other protective bioactive molecules in mucus include trefoil factors involved in the repair and restitution of the epithelial mucosa (Taupin and Podolsky, 2003), and resistin-like molecule $\beta(\operatorname{Re} \operatorname{lm} \beta$ ) which contribute to the barrier properties of mucus by enhancing mucin gene expression and preventing bacteria from breaching the mucus layer (Hogan et al., 2006; Krimi et al., 2008). In general, mucus protects underlying epithelial cells from chemical, microbial, enzymatic, and mechanical damage.

The main component of mucus, mucin glycoproteins, are heavily $o$-glycosylated in large peptide domains harboring repeats of the amino acids serine and threonine. These areas are known as variable number tandem repeat (VNTR) regions and vary in length both between individuals and different mucin types, leading to VNTR polymorphisms. Carbohydrates constitute approximately $70 \%$ of the mass of mucin glycoproteins, conferring mucins with water holding capacity, rigidity, protease resistance, and a high charge density (Klein et al., 1993; Moncada et al., 2003). Up to 16 human mucins have been described, falling into three subfamilies: secreted mucins (MUC7), cell surface mucins (MUC1,
MUC4, MUC3A/B, MUC12, MUC13, MUC15, MUC16, MUC 17, MUC20), and gel-forming mucins (MUC2, MUC5AC, MUC5B, MUC6, and MUC19). A cluster of four genes on chromosome 11 encode gel-forming mucins, which are the main mucin subfamily in mucus and confer its visco-elastic properties. Cysteine residues at the $\mathrm{N}$ - and $\mathrm{C}$-termini of gel-forming mucins form intermolecular and intramolecular disulfide bonds, leading to homo-oligomerization of mucins in regions known as von Willebrand factor-like or D domains (Moncada et al., 2003; Linden et al., 2008b). In the gastrointestinal tract, mucin types are differentially expressed. Mucin sub-types in the colon include MUC2, MUC1, MUC3A/B, MUC4, MUC12, MUC13, MUC15, MUC17, and MUC20. The stomach contains MUC5AC, MUC6, MUC1, MUC4, MUC12, MUC13, and MUC17, while small intestinal mucins include MUC2, MUC3A/B, MUC12, MUC13, MUC15, and MUC17 (Moran et al., 2011).

\section{MUCUS AND ENTERIC BACTERIAL PATHOGENS}

Enteric pathogens have to colonize and circumvent the mucus layer prior to causing disease. To establish infection, these bacteria have to bypass both physical and immunological barriers in the gastrointestinal tract. The visco-elastic mucus gel layer protects underlying epithelial cells by trapping pathogens in the loose mucus and clearing them both during gut movements and mucus turnover. In addition, mucin glycoproteins bind and retard bacteria in mucus, preventing them from accessing epithelial cells. Furthermore, antimicrobial peptides present in mucus and gut microflora are directly antagonistic to pathogens. Intestinal microbiota also competitively exclude gut pathogens by occupying mucin receptors, hence preventing pathogen access to the mucosa (reviewed in Linden et al., 2008b). 
Enteric pathogens typically breach the mucus layer by employing mucus degradative enzymes. Mucinases, sialidases, $N$-acetyl neuraminate lyases, glycosulfatases, and sialate $o$-acetylesterases are some of the mucinolytic enzymes employed by gut pathogens (Corfield et al., 1992, 1993; Haider et al., 1993; Homer et al., 1994). For example Vibrio cholerae expresses HapA which has both mucinolytic and cytotoxic activity (Silva et al., 2003). In addition, chemotaxis and flagellar motility facilitate colonization and penetration of the mucus barrier by enteric pathogens (discussed below for Campylobacter jejuni).

In response to the presence of gut microbes, mucin synthesis and glycosylation are regulated. Adherence by probiotic bacteria and products from some bacteria increase the synthesis of mucins from epithelial cells (Smirnova et al., 2003). For example, colonic Mucl expression is upregulated in both mouse and human intestinal bacterial infections (Linden et al., 2008a). Furthermore, mucin glycosylation changes have been documented following Helicobacter pylori infection (reviewed in Moran et al., 2011) and H. pylori lipopolysaccharide (LPS) inhibits mucin synthesis in gastric epithelial cells (Slomiany and Slomiany, 2006). $H$. pylori and the stomach gel-forming mucin MUC5AC have been shown to colocalize, and these bacteria bind the dimeric form of the human recombinant trefoil factor 1 (TFF1; Van den Brink et al., 2000; Clyne et al., 2004). Recently, it has been shown that H. pylori binds TFF1 by means of its rough form LPS in a $\mathrm{pH}$ dependent manner (Reeves et al., 2008). The mechanism of bacterial LPS binding to TFF1 is not known. However, TFF1 harbors a hydrophobic patch of solvent-exposed residues on its surface which may act as a possible carbohydrate binding domain (Reeves et al., 2008). In addition, a pH of between 5 and 6 was found to be optimal for the interaction between TFF1 and H. pylori. This $\mathrm{pH}$ range is typical of that found in the mucosal niche occupied by $H$. pylori in vivo supporting the possibility that $H$. pylori binding to TFF1 may promote bacterial colonization in the stomach (Schreiber et al., 2004; Reeves et al., 2008).

From the above it is clear that while on the one hand components of mucus influence bacterial colonization and virulence properties, on the other, the presence of bacteria has a marked effect on mucin expression. Therefore, while the mucus layer and its constituents form part of the innate defense against pathogens, mucosal organisms have evolved remarkable strategies to circumvent (or even subvert for their own advantage) this barrier.

\section{THE INTERACTION OF C. JEJUNI WITH MUCUS AND MUCINS MUCUS AND MUCINS AS A BARRIER IN C. JEJUNI INFECTION}

Much of our understanding of how intestinal mucins protect the mucosa from infection with C. jejuni has evolved only recently, led by groundbreaking work from McGuckin and colleagues at the University of Queensland (McAuley et al., 2007; Linden et al., 2008a,b; Dawson et al., 2009). The cell surface mucin, Muc1, was found to be upregulated following infection with both the natural mouse pathogen Citrobacter rodentium and with C. jejuni (McAuley et al., 2007; Linden et al., 2008a). Muc1 negative mice were shown to have a marked increase in translocation of C. jejuni to systemic organs. In addition Mucl is upregulated in human colonic biopsies following naturally occurring C. jejuni infection
(Linden et al., 2008a). A not dissimilar role for mucin sulfation in the protection of mice from C. jejuni systemic infection (a marker of intestinal barrier function) subsequently was demonstrated using a mouse Nas1 knockout model (Dawson et al., 2009). Interestingly, while Mucl also has a role in protecting mice from $H$. pylori infection, it does not help protect mice from the pathologic effects of $H$. felis infection (Every et al., 2008). From these experiments, it is obvious that the mucus barrier has evolved a complex array of protective mechanisms against infection and these appear not to be simply generic antibacterial barriers. Nonetheless, it will be important to attempt to document the role of these mucus characteristics in Campylobacter models that approximate more closely those of human infection, a problem that continues to bedevil the wider field of Campylobacter pathogenesis.

\section{ADAPTATIONS OF $\boldsymbol{C}$. JEJUNI TO LIFE IN THE MUCUS LAYER}

Ultimately, C. jejuni is very successful in overcoming the intricate barrier structures posed by mucus and mucins in order to establish enteric infection, at least in the human intestinal tract. While the exact details of how C. jejuni establishes infection remain elusive, the complex interplay between bacterium and host mucosal surface has been the subject of intensive research in recent years and an understanding is beginning to emerge of the role of mucus in that relationship.

\section{Motility/chemotaxis}

Flagellar motility and chemotaxis toward components of mucus are thought to be crucial in the life cycle of C. jejuni, both as a commensal and as a pathogen. Virulence in the ferret model, host colonization, adhesion, and invasion all require flagellar motility (Young et al., 2007). Chemotaxis receptors together with other elements of the C. jejuni chemotaxis and motility machinery are known to be required for colonization in a chick model of infection (Hendrixson and DiRita, 2004). Likewise, flagellar genes and regulators of flagellar biosynthesis such as FlgR, $\sigma^{28}$, and $\sigma^{54}$ are all required for chick colonization (Hendrixson, 2006). Chemoattractants for C. jejuni include amino acids such as L-glutamate, L-serine, L-cysteine, L-aspartate, and the sugar fucose (Hugdahl et al., 1988; Szymanski et al., 1999).

In solutions of increased viscosity comparable to mucus, $C$. jejuni is highly motile, demonstrating peaks of motility with longer path lengths (Ferrero and Lee, 1988; Szymanski et al., 1995). The corkscrew-like shape of these bacteria and the relatively short O-side chains of its lipooligosaccharide (LOS) are purported to prevent non-specific binding of C. jejuni to mucin glycoproteins (McSweegan and Walker, 1986). This suggests an adaptation of these bacteria to the environment of mucus, thus conferring $C$. jejuni with an ecological advantage while in mucus (Ferrero and Lee, 1988). Increased motility of C. jejuni in highly viscous solutions correlates with increased virulence in vitro. Pre-incubation of C. jejuni in media of increased viscosity increases bacterial binding and internalization into Caco-2 cells (Szymanski et al., 1995).

\section{Reproduction in mucus}

Campylobacter jejuni has been shown to reproduce in chicken mucus (Van Deun et al., 2008). Van Deun et al. showed that $C$. jejuni multiplies in PBS supplemented with mucus but not in 
PBS alone or chicken cecal contents. In agreement with these findings, we have recently demonstrated $C$. jejuni replication in growth medium supplemented with mucus derived from a mucus-adherent subclone of methotrexate treated HT29 cells, HT29MTXE12 (E12; Alemka et al., 2010a). We also showed that C. jejuni reproduced following colonization of E12 cells (with mucus), compared with HT29 cells that lacked a mucus layer. Taken together, these studies indicate that C. jejuni grows and thrives in the mucus milieu, an adaptation that likely helps to ensure its persistence in the host.

\section{Adhesion/invasion and pathogenicity}

Conventional tissue culture has contributed greatly to our understanding of how enteric pathogens such as C. jejuni elaborate disease. For instance, infection studies with intestinal epithelial cells led to the identification of $C$. jejuni adhesins such as CadF. CadF, also required for chick colonization (Ziprin et al., 1999), binds fibronectin located at the basolateral aspects of cells, and facilitates bacterial internalization (Monteville et al., 2003; KrauseGruszczynska et al., 2007). Likewise, JlpA, a lipoprotein on the surface of C. jejuni, interacts with heat shock protein $90 \alpha$ leading to the activation of $\mathrm{p} 38$ mitogen activated protein kinases (MAPK) and NF- $\kappa \mathrm{B}$, thereby eliciting pro-inflammatory responses in Hep2 cells (Jin et al., 2003). In addition, both microtubules ( $\mathrm{Hu}$ and Kopecko, 1999) and the actin cytoskeleton (Monteville et al., 2003; Krause-Gruszczynska et al., 2007) are involved in C. jejuni invasion. Furthermore, these bacteria translocate cultured intestinal epithelial cells by migrating through both the paracellular (Monteville and Konkel, 2002) and transcellular routes (Bras and Ketley, 1999; Hu et al., 2008).

Despite the utility of conventional cell lines, the absence of an adherent mucus layer on these models is a significant weakness. In order to overcome this, we have attempted to better understand the role of mucus in infection by characterizing the interaction of C. jejuni with E12 cells (Behrens et al., 2001; Alemka et al., 2010a). E12 cells were first validated as an appropriate in vitro model of Campylobacter-host interactions by demonstrating mucus colonization (Figure 1), adhesion, invasion, and translocation to the basolateral aspects of cells. Compared with parental HT29 cells that lack a mucus layer, there was enhanced bacterial binding and internalization into E12 cells (Alemka et al., 2010a). Combined with previous studies (de Melo and Pechere, 1988; Szymanski

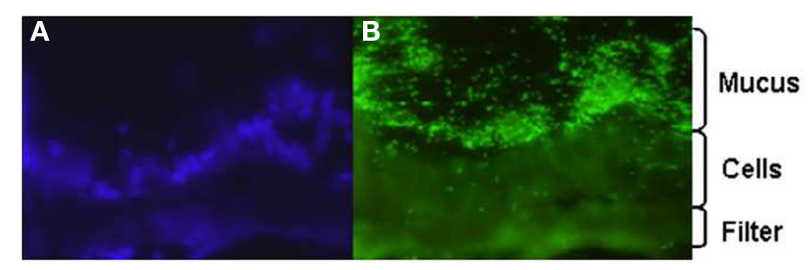

FIGURE 1 | Fluorescence microscopy image of $\boldsymbol{C}$. jejuni colonization of E12 mucus. E12 cells were grown for 14 days in transwell inserts and infected with $C$. jejuni. The filter was excised and wrapped between two pieces of frozen liver and sections probed with DAPI (A) to reveal cell nuclei and (B) anti-Campylobacter antibodies. Mag: $\times 400$. et al., 1995; Byrne et al., 2007) these findings indicate that C. jejuni not only has evolved mechanisms to breach the protective mucus barrier layer, but also appears to have subverted the mucus to enhance pathogenicity. In support of this concept, the presence of MUC2 (the main intestinal gel-forming mucin) has been shown to upregulate a number of $C$. jejuni virulence genes, including those involved in motility $(f l a A)$, adhesion $(j l p A)$, invasion $(c i a B)$, and toxin formation ( $c d t$; Tu et al., 2008).

Which component or characteristic of mucus and mucin that modulates C. jejuni pathogenicity is not known. However, recent studies have suggested some intriguing possibilities. It has long been known that $C$. jejuni does not metabolize glucose due to the absence of the enzyme phosphofructokinase in its genome (Parkhill et al., 2000; Guccione et al., 2008) and, because of the absence of genes involved in sugar metabolism (Parkhill et al., 2000; Muraoka and Zhang, 2011), it has generally been assumed that this organism is unable to use any form of carbohydrate as a substrate. Rather these bacteria use amino acids such as glutamate, asparagine, proline, serine, and aspartate as a source of carbon (Guccione et al., 2008; Hofreuter et al., 2008).

However, C. jejuni was recently shown to metabolize fucose (Muraoka and Zhang, 2011; Stahl et al., 2011). In these studies, growth of C. jejuni was enhanced in media supplemented with fucose. Several strains of $C$. jejuni now have been shown to possess a genomic island that is linked with the ability to take up and utilize fucose as a source of carbon (Stahl et al., 2011). In light of the fact that Campylobacter displays chemotaxis to mucin (which harbors fucosylated glycans), binds to mucin (Sylvester et al., 1996), and the finding that these bacteria reproduce in both human and chicken mucus (Van Deun et al., 2008; Alemka et al., 2010a), it is tempting to speculate that $C$. jejuni acquires and utilizes constituents of mucus to sustain its commensal and pathogenic modes of existence, and that differences in the composition of mucus across species may contribute to C. jejuni pathogenicity in humans as opposed to commensalism in other animals (discussed below). Furthermore, the importance of the interaction of C. jejuni with fucosylated structures possibly could be exploited as a novel antimicrobial therapy in C. jejuni infections (Ruiz-Palacios et al., 2003).

\section{Influence of gut microbiota in the mucus layer}

In the gut, microbiota are known to colonize the outer loose mucus layer (Johansson et al., 2008). It is thought that microbiota protect the gut from invading enteric pathogens by competing for nutrients and mucosal binding sites with pathogens, by modulating the mucosal immune response and also through direct antagonism (Neish, 2009). Many commensal derived bacteria have been proposed as potential anti infective probiotics. On this basis, we investigated the effect of probiotic colonization of the mucus of E12 cells on C. jejuni pathogenicity (Alemka et al., 2010a). Individual probiotic strains (Lactobacillus rhamnosus, L. salivarius, Bifidobacterium longum), or cocktails of these probiotics, reduced C. jejuni colonization, invasion, and translocation of mucus-adherent E12 cells in a strain dependent manner. Pre-colonization of E12 mucus with probiotics was required to attenuate $C$. jejuni virulence, suggesting that probiotics have a potential as prophylactic agents against C. jejuni infections. The potential use of probiotics to reduce 
Campylobacter numbers in chickens and thereby prevent zoonotic transmission may be a particularly attractive strategy to reduce the burden of illness in humans.

\section{CHICKEN MUCUS AND CAMPYLOBACTER COMMENSALISM}

The consumption of contaminated poultry is the principal mode of transmission of $C$. jejuni to humans. Up to $100 \%$ of point-ofsale chicken has been shown to be contaminated with C. jejuni (Madden et al., 1998; Pearson et al., 2000). Despite heavy colonization (up to $10^{10}$ colony forming units per gram of infected intestine) in chickens, C. jejuni does not cause disease. These bacteria avidly colonize the mucus layer of deep cecal crypts, but do not adhere to or invade chicken intestinal epithelial cells (reviewed in Young et al., 2007).

Why C. jejuni is pathogenic in the human population but establishes a commensal mode of existence in chickens is uncertain. An obvious difference between humans and chickens is body temperature. The human body temperature is $37^{\circ} \mathrm{C}$ while it ranges from 41 to $45^{\circ} \mathrm{C}$ in chickens. Campylobacters are therefore adapted to higher temperatures in chickens but the effect of temperature on the pathogenicity of C. jejuni is not yet fully understood. The two-component system RacRS has been implicated as possibly influencing the adaptation of C. jejuni to temperature. Mutants in this regulatory pathway show defects in viability at $42^{\circ} \mathrm{C}$, suggesting that it may contribute to the survival and growth of C. jejuni at higher temperatures (MacKichan et al., 2004). In addition, differences in Campylobacter gene expression have been reported following growth at $37^{\circ} \mathrm{C}$ compared with $42^{\circ} \mathrm{C}$. In one such study (Stintzi, 2003), at $42^{\circ} \mathrm{C}$, there was an increase in the expression of chemotaxis and motility genes and an overall $20 \%$ general difference in gene expression compared with $37^{\circ} \mathrm{C}$. Therefore, body temperature may influence the commensal versus pathogenic lifestyles of C. jejuni. However, temperature alone is unlikely to account for the difference in clinical manifestation across species.

We originally hypothesized that species-specific tissue tropism might contribute to the differences in clinical outcome of Campylobacter infection in humans and chickens. However, we (Byrne et al., 2007) and others (Van Deun et al., 2008) subsequently showed that C. jejuni both adheres to and invades chicken primary intestinal cells. These data indicate that Campylobacter commensalism in chickens as opposed to pathogenicity in humans is not due to lack of the relevant adhesin-receptor interactions that mediate invasiveness in the chicken intestinal epithelium. In light of these findings we investigated the possibility that intestinal mucus contributes to the inhibitory effect of the chicken intestinal milieu on C. jejuni virulence. We demonstrated that crude chicken intestinal mucus, markedly attenuates $C$. jejuni binding and internalization (Byrne et al., 2007). Consistent with these findings, mucus colonization of the human mucus-adherent E12 cell line increased C. jejuni adhesion and invasion of E12 cells (Alemka et al., 2010a). Subsequently we demonstrated that purified chicken intestinal mucins, when pre-incubated with C. jejuni, preferentially attenuate bacterial internalization into HCT-8 cells (Figure 2). The effect depends on the region of the chicken gut from where mucins are isolated (Alemka et al., 2010b). In the same study, attenuation of bacterial invasion by chicken mucins appeared to have been mediated by mucin glycans, since sodium metaperiodate treatment of chicken mucins abolished the attenuating effect of purified mucins on bacterial internalization. Intriguingly, removal of terminal fucose or sialic acid residues failed to inhibit the effect of chicken mucin on C. jejuni invasion. However, as chicken mucin glycoproteins are heavily sulfated compared with human mucins (unpublished observations), sulfation may have limited the activity of the fucosidase and sialidase enzymes used in our study (Alemka et al., 2010b). Conversely, it is possible that sulfation of mucin oligosaccharides may in itself influence $C$. jejuni mucus colonization and pathogenicity across species. Taken together, these data indicate that mucus and mucins modulate $C$. jejuni virulence properties and may contribute to the differential clinical manifestations exhibited by $C$. jejuni in different hosts (Figure 3).
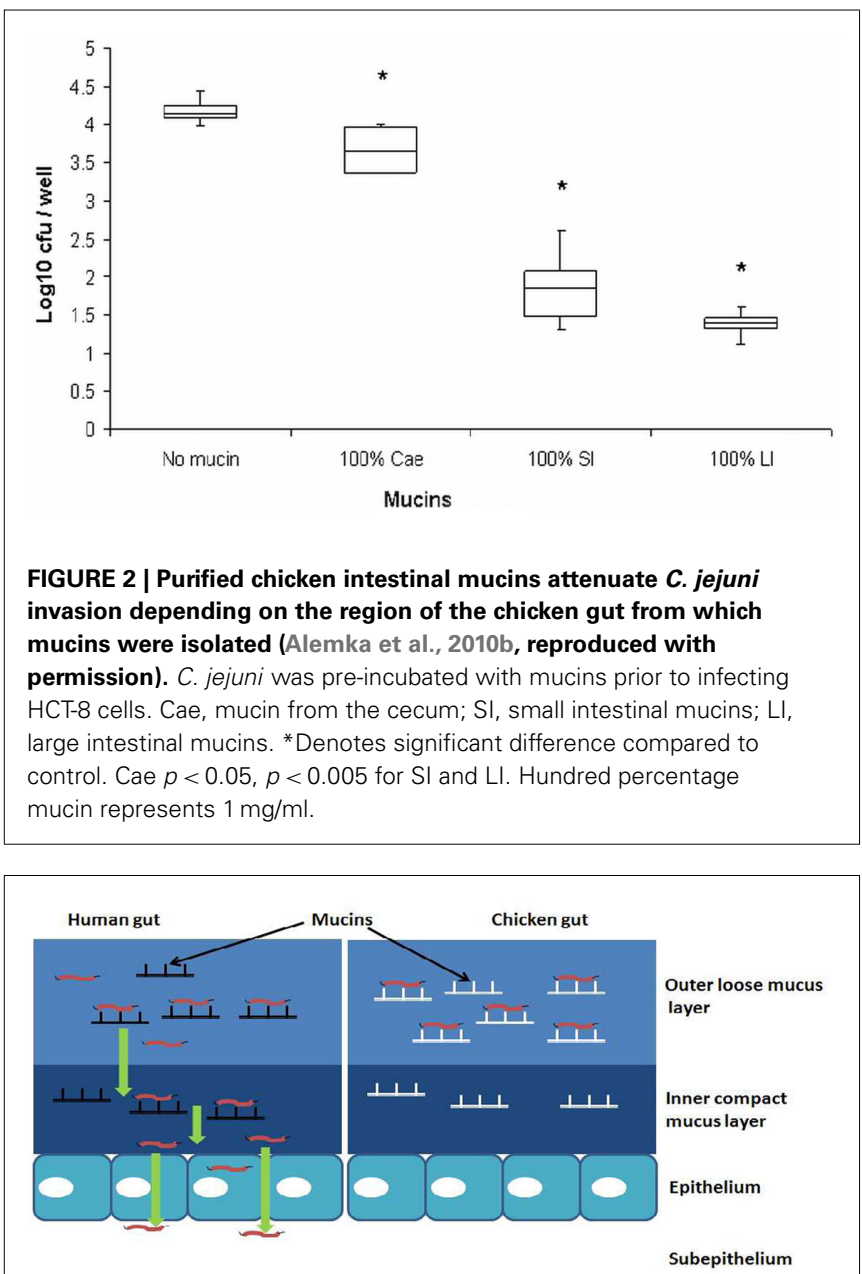

FIGURE 3 | Hypothetical model of how mucus and mucins modulate the pathogenicity of $\boldsymbol{C}$. jejuni in a species-specific manner: in mucus C. jejuni displays chemotaxis and flagellar motility toward components of mucin. The characteristic spiral shape and the ability of $C$. jejuni to replicate in mucus suggests an adaptation of these bacteria to the mucus milieu. Interaction with human mucin enhances bacterial binding and internalization into underlying epithelial cells. In chickens, mucin glycoproteins prevent $C$. jejuni association with intestinal epithelial cells, possibly by interfering with their transit through the inner mucus layer and/or onto the appropriate host cell receptors. 


\section{CONCLUSION AND FUTURE PERSPECTIVES}

The visco-elastic mucus gel layer is part of the innate defense mechanism against infecting bacteria. Mucus colonization by enteric pathogens influences the outcome of infection with these bacteria employing a variety of mechanisms to circumvent the mucus layer and gain access to underlying epithelial cells. C. jejuni is attracted to and metabolizes components of mucus. These bacteria are highly motile with a characteristic spiral shape and corkscrew-like motility that suggests an adaptation to the environment of mucus. Recent studies indicate that mucus and mucins

\section{REFERENCES}

Alemka, A., Clyne, M., Shanahan, F., Tompkins, T., Corcionivoschi, N., and Bourke, B. (2010a). Probiotic colonization of the adherent mucus layer of HT29MTXE12 cells attenuates Campylobacter jejuni virulence properties. Infect. Immun. 78, 2812-2822.

Alemka, A., Whelan, S., Gough, R., Clyne, M., Gallagher, M. E., Carrington, S. D., and Bourke, B. (2010b). Purified chicken intestinal mucin attenuates Campylobacter jejuni pathogenicity in vitro. J. Med. Microbiol. 59, 898-903.

Behrens, I., Stenberg, P., Artursson, P., and Kissel, T. (2001). Transport of lipophilic drug molecules in a new mucus-secreting cell culture model based on HT29-MTX cells. Pharm. Res. 18, 1138-1145.

Bras, A. M., and Ketley, J. M. (1999). Transcellular translocation of Campylobacter jejuni across human polarised epithelial monolayers. FEMS Microbiol. Lett. 179, 209-215.

Byrne, C. M., Clyne, M., and Bourke, B. (2007). Campylobacter jejuni adhere to and invade chicken intestinal epithelial cells in vitro. Microbiology 153, 561-569.

Clyne, M., Dillon, P., Daly, S., O’Kennedy, R., May, F. E., Westley, B. R., and Drumm, B. (2004). Helicobacter pylori interacts with the human single-domain trefoil protein TFF1. Proc. Natl. Acad. Sci. U.S.A. 101, 7409-7414.

Corfield, A. P., Wagner, S. A., Clamp, J. R., Kriaris, M. S., and Hoskins, L. C. (1992). Mucin degradation in the human colon: production of sialidase, sialate $\mathrm{O}$ acetylesterase, $\mathrm{N}$-acetylneuraminate lyase, arylesterase, and glycosulfatase activities by strains of fecal bacteria. Infect. Immun. 60, 3971-3978.

Corfield, A. P., Wagner, S. A., O’Donnell, L. J., Durdey, P., Mountford, R. A., and Clamp, J. R. (1993). The roles of enteric bacterial sialidase, sialate
$\mathrm{O}$-acetyl esterase and glycosulfatase in the degradation of human colonic mucin. Glycoconj. J. 10, 72-81.

Dawson, P. A., Huxley, S., Gardiner, B., Tran, T., McAuley, J. L., Grimmond, S., McGuckin, M. A., and Markovich, D. (2009). Reduced mucin sulfonation and impaired intestinal barrier function in the hyposulfataemic NaS1 null mouse. Gut 58, 910-919.

de Melo, M. A., and Pechere, J. C. (1988). Effect of mucin on Campylobacter jejuni association and invasion on HEp-2 cells. Microb. Pathog. 5, 71-76.

Every, A. L., Chionh, Y. T., Skene, C. D., McGuckin, M. A., and Sutton, P. (2008). Muc1 limits Helicobacter felis binding to gastric epithelial cells but does not limit colonization and gastric pathology following infection. Helicobacter 13, 489-493.

Ferrero, R. L., and Lee, A. (1988). Motility of Campylobacter jejuni in a viscous environment: comparison with conventional rod-shaped bacteria. J. Gen. Microbiol. 134, 53-59.

Guccione, E., Leon-Kempis Mdel, R., Pearson, B. M., Hitchin, E., Mulholland, F., Van Diemen, P. M., Stevens, M. P., and Kelly, D. J. (2008). Amino acid-dependent growth of Campylobacter jejuni: key roles for aspartase (AspA) under microaerobic and oxygen-limited conditions and identification of AspB (Cj0762), essential for growth on glutamate. Mol. Microbiol. 69, 77-93.

Haider, K., Hossain, A., Wanke, C., Qadri, F., Ali, S., and Nahar, S. (1993). Production of mucinase and neuraminidase and binding of Shigella to intestinal mucin. J. Diarrhoeal Dis. Res. 11, 88-92.

Hendrixson, D. R. (2006). A phasevariable mechanism controlling the Campylobacter jejuni FlgR response regulator influences commensalism. Mol. Microbiol. 61, 1646-1659.

Hendrixson, D. R., and DiRita, V. J. (2004). Identification of Campylobacter jejuni genes involved in commensal colonization of the

influence $C$. jejuni in a species-specific manner with chicken mucins attenuating $C$. jejuni pathogenicity. Therefore, differences in the composition of mucus and mucins across species appear to contribute to $C$. jejuni disease causing lifestyle in the human population, as opposed to commensalism in chickens. Increased knowledge and understanding of the interaction of Campylobacter with mucus and mucins has the potential to lead to the development of intervention strategies that take advantage of the propensity of these bacteria to inhabit the intriguing supramucosal niche that is the mucus layer.

chick gastrointestinal tract. $\mathrm{Mol}$. Microbiol. 52, 471-484.

Hofreuter, D., Novik, V., and Galan, J. E. (2008). Metabolic diversity in Campylobacter jejuni enhances specific tissue colonization. Cell Host Microbe 4, 425-433.

Hogan, S. P., Seidu, L., Blanchard, C. Groschwitz, K., Mishra, A., Karow, M. L., Ahrens, R., Artis, D., Murphy, A. J., Valenzuela, D. M., Yancopoulos, G. D., and Rothenberg, M. E. (2006). Resistin-like molecule beta regulates innate colonic function: barrier integrity and inflammation susceptibility. J. Allergy Clin. Immunol. 118, 257-268.

Homer, K. A., Whiley, R. A., and Beighton, D. (1994). Production of specific glycosidase activities by Streptococcus intermedius strain UNS35 grown in the presence of mucin. J. Med. Microbiol. 41, 184-190.

Hu, L., and Kopecko, D. J. (1999). Campylobacter jejuni 81-176 associates with microtubules and dynein during invasion of human intestinal cells. Infect. Immun. 67, 4171-4182.

Hu, L., Tall, B. D., Curtis, S. K., and Kopecko, D. J. (2008). Enhanced microscopic definition of Campylobacter jejuni 81-176 adherence to, invasion of, translocation across, and exocytosis from polarized human intestinal Caco-2 cells. Infect. Immun. 76, 5294-5304.

Hugdahl, M. B., Beery, J. T., and Doyle, M. P. (1988). Chemotactic behavior of Campylobacter jejuni. Infect. Immun. 56, 1560-1566.

Jin, S., Song, Y. C., Emili, A., Sherman, P. M., and Chan, V. L. (2003). JlpA of Campylobacter jejuni interacts with surface-exposed heat shock protein 90alpha and triggers signalling pathways leading to the activation of NF-kappaB and p38 MAP kinase in epithelial cells. Cell. Microbiol. 5 , 165-174.

Johansson, M. E., Phillipson, M. Petersson, J., Velcich, A., Holm, L., and Hansson, G. C. (2008).
The inner of the two Muc2 mucin-dependent mucus layers in colon is devoid of bacteria. Proc. Natl. Acad. Sci. U.S.A. 105, 15064-15069.

Klein, A., Carnoy, C., Lamblin, G., Roussel, P., Van Kuik, J. A., and Vliegenthart, J. F. (1993). Isolation and structural characterization of novel sialylated oligosaccharidealditols from respiratory-mucus glycoproteins of a patient suffering from bronchiectasis. Eur. J. Biochem. 211, 491-500.

Krause-Gruszczynska, M., Rohde, M., Hartig, R., Genth, H., Schmidt, G., Keo, T., Konig, W., Miller, W. G., Konkel, M. E., and Backert, S. (2007). Role of the small Rho GTPases Racl and Cdc42 in host cell invasion of Campylobacter jejuni. Cell. Microbiol. 9, 2431-2444.

Krimi, R. B., Kotelevets, L., Dubuquoy, L., Plaisancie, P., Walker, F., Lehy, T., Desreumaux, P., Van Seuningen, I., Chastre, E., Forgue-Lafitte, M. E., and Marie, J. C. (2008). Resistin-like molecule beta regulates intestinal mucous secretion and curtails TNBS-induced colitis in mice. Inflamm. Bowel Dis. 14, 931-941.

Linden, S. K., Florin, T. H., and McGuckin, M. A. (2008a). Mucin dynamics in intestinal bacterial infection. PLoS ONE 3, e3952. doi:10.1371/journal.pone.0003952

Linden, S. K., Sutton, P., Karlsson, N. G., Korolik, V., and McGuckin, M. A (2008b). Mucins in the mucosal barrier to infection. Mucosal Immunol. 1, 183-197.

MacKichan, J. K., Gaynor, E. C., Chang, C., Cawthraw, S., Newell, D. G., Miller, J. F., and Falkow, S. (2004) The Campylobacter jejuni dccRS two-component system is required for optimal in vivo colonization but is dispensable for in vitro growth. Mol. Microbiol. 54, 1269-1286.

Madden, R. H., Moran, L., and Scates, P. (1998). Frequency of occurrence of Campylobacter spp. in red meats and poultry in Northern Ireland 
and their subsequent subtyping using polymerase chain reactionrestriction fragment length polymorphism and the random amplified polymorphic DNA method. $J$. Appl. Microbiol. 84, 703-708.

McAuley, J. L., Linden, S. K., Png, C. W., King, R. M., Pennington, H. L., Gendler, S. J., Florin, T. H., Hill, G. R., Korolik, V., and McGuckin, M. A. (2007). MUC1 cell surface mucin is a critical element of the mucosal barrier to infection. J. Clin. Invest. 117, 2313-2324.

McGuckin, M. A., Linden, S. K., Sutton, P., and Florin, T. H. (2011). Mucin dynamics and enteric pathogens. Nat. Rev. Microbiol. 9, 265-278.

McSweegan, E., and Walker, R. I. (1986). Identification and characterization of two Campylobacter jejuni adhesins for cellular and mucous substrates. Infect. Immun. 53, 141-148.

Moncada, D. M., Kammanadiminti, S. J., and Chadee, K. (2003). Mucin and Toll-like receptors in host defense against intestinal parasites. Trends Parasitol. 19, 305-311.

Monteville, M. R., and Konkel, M. E. (2002). Fibronectin-facilitated invasion of T84 eukaryotic cells by Campylobacter jejuni occurs preferentially at the basolateral cell surface. Infect. Immun. 70, 6665-6671.

Monteville, M. R., Yoon, J. E., and Konkel, M. E. (2003). Maximal adherence and invasion of INT 407 cells by Campylobacter jejuni requires the CadF outer-membrane protein and microfilament reorganization. Microbiology 149, 153-165.

Moran, A. P., Gupta, A., and Joshi, L. (2011). Sweet-talk: role of host glycosylation in bacterial pathogenesis of the gastrointestinal tract. Gut 60, 1412-1425.

Muraoka, W. T., and Zhang, Q. (2011). Phenotypic and genotypic evidence for L-fucose utilization by Campylobacter jejuni. J. Bacteriol. 193, 1065-1075.
Neish, A. S. (2009). Microbes in gastrointestinal health and disease. Gastroenterology 136, 65-80.

Parkhill, J., Wren, B. W., Mungall, K., Ketley, J. M., Churcher, C., Basham, D., Chillingworth, T., Davies, R. M., Feltwell, T., Holroyd, S., Jagels, K., Karlyshev, A. V., Moule, S., Pallen, M. J., Penn, C. W., Quail, M. A., Rajandream, M. A., Rutherford, K. M., Van Vliet, A. H., Whitehead, S., and Barrell, B. G. (2000). The genome sequence of the food-borne pathogen Campylobacter jejuni reveals hypervariable sequences. Nature 403, 665-668.

Pearson, A. D., Greenwood, M. H., Donaldson, J., Healing, T. D., Jones, D. M., Shahamat, M., Feltham, R. K., and Colwell, R. R. (2000). Continuous source outbreak of campylobacteriosis traced to chicken. J. Food Prot. 63, 309-314.

Reeves, E. P., Ali, T., Leonard, P., Hearty, S., O'Kennedy, R., May, F. E., Westley, B. R., Josenhans, C., Rust, M., Suerbaum, S., Smith, A., Drumm, B., and Clyne, M. (2008). Helicobacter pylori lipopolysaccharide interacts with TFF1 in a $\mathrm{pH}$ dependent manner. Gastroenterology 135, 2043-2054.

Ruiz-Palacios, G. M., Cervantes, L. E., Ramos, P., Chavez-Munguia, B., and Newburg, D. S. (2003). Campylobacter jejuni binds intestinal $\mathrm{H}(\mathrm{O})$ antigen (Fuc alpha 1, 2Gal beta 1, 4GlcNAc), and fucosyl oligosaccharides of human milk inhibit its binding and infection. J. Biol. Chem. 278, 14112-14120.

Schreiber, S., Konradt, M., Groll, C., Scheid, P., Hanauer, G., Werling, H. O., Josenhans, C., and Suerbaum, S. (2004). The spatial orientation of Helicobacter pylori in the gastric mucus. Proc. Natl. Acad. Sci. U.S.A. 101, 5024-5029.

Silva, A. J., Pham, K., and Benitez, J. A. (2003). Haemagglutinin/protease expression and mucin gel penetration in $\mathrm{El}$ Tor biotype
Vibrio cholerae. Microbiology 149, 1883-1891.

Slomiany, B. L., and Slomiany, A. (2006). Cytosolic phospholipase A2 activation in Helicobacter pylor lipopolysaccharide-induced interference with gastric mucin synthesis. IUBMB Life 58, 217-223.

Smirnova, M. G., Guo, L., Birchall, J. P., and Pearson, J. P. (2003). LPS up-regulates mucin and cytokine mRNA expression and stimulates mucin and cytokine secretion in goblet cells. Cell. Immunol. 221, 42-49.

Stahl, M., Friis, L. M., Nothaft, H., Liu, X., Li, J., Szymanski, C. M., and Stintzi, A. (2011). L-fucose utilization provides Campylobacter jejuni with a competitive advantage. Proc. Natl. Acad. Sci. U.S.A. 108, 7194-7199.

Stintzi, A. (2003). Gene expression profile of Campylobacter jejuni in response to growth temperature variation. J. Bacteriol. 185 , 2009-2016.

Sylvester, F. A., Philpott, D., Gold, B., Lastovica, A., and Forstner, J. F. (1996). Adherence to lipids and intestinal mucin by a recently recognized human pathogen, Campylobacter upsaliensis. Infect. Immun. 64, 4060-4066.

Szymanski, C. M., King, M., Haardt, M., and Armstrong, G. D. (1995). Campylobacter jejuni motility and invasion of Caco-2 cells. Infect. Immun. 63, 4295-4300.

Szymanski, C. M., Yao, R., Ewing, C. P., Trust, T. J., and Guerry, P. (1999). Evidence for a system of general protein glycosylation in Campylobacter jejuni. Mol. Microbiol. 32, 1022-1030.

Taupin, D., and Podolsky, D. K. (2003). Trefoil factors: initiators of mucosal healing. Nat. Rev. Mol. Cell Biol. 4, 721-732.

Tu, Q. V., McGuckin, M. A., and Mendz, G. L. (2008). Campylobacter jejuni response to human mucin MUC2: modulation of colonization and pathogenicity determinants. $J$. Med. Microbiol. 57, 795-802.

Van den Brink, G. R., Tytgat, K. M., Van Der Hulst, R. W., Van Der Loos, C. M., Einerhand, A. W., Buller, H. A., and Dekker, J. (2000). H. pylori colocalises with MUC5AC in the human stomach. Gut 46, 601-607.

Van Deun, K., Pasmans, F., Ducatelle, R., Flahou, B., Vissenberg, K., Martel, A., Van Den Broeck, W., Van Immerseel F., and Haesebrouck, F. (2008). Colonization strategy of Campylobacter jejuni results in persistent infection of the chicken gut. Vet. Microbiol. 130, 285-297.

Young, K. T., Davis, L. M., and Dirita, V. J. (2007). Campylobacter jejuni: molecular biology and pathogenesis Nat. Rev. Microbiol. 5, 665-679.

Ziprin, R. L., Young, C. R., Stanker, L. H., Hume, M. E., and Konkel, M. E. (1999). The absence of cecal colonization of chicks by a mutant of Campylobacter jejuni not expressing bacterial fibronectin-binding protein. Avian Dis. 43, 586-589.

Conflict of Interest Statement: The authors declare that the research was conducted in the absence of any commercial or financial relationships that could be construed as a potential conflict of interest.

Received: 30 October 2011; accepted: 03 February 2012; published online: $20 \mathrm{Feb}$ ruary 2012.

Citation: Alemka A, Corcionivoschi N and Bourke B (2012) Defense and adaptation: the complex inter-relationship between Campylobacter jejuni and mucus. Front. Cell. Inf. Microbio. 2:15. doi: 10.3389/fcimb.2012.00015 Copyright (C) 2012 Alemka, Corcionivoschi and Bourke. This is an open-access article distributed under the terms of the Creative Commons Attribution Non Commercial License, which permits non-commercial use, distribution, and reproduction in other forums, provided the original authors and source are credited. 